Research articles

\title{
Manipulating the magnetic anisotropy and magnetization dynamics by stress: Numerical calculation and experiment
}

\author{
M.A. Correa*, F. Bohn \\ Departamento de Física, Universidade Federal do Rio Grande do Norte, 59078-900 Natal, RN, Brazil
}

\section{A R T I C L E I N F O}

\section{Article history:}

Received 3 August 2017

Received in revised form 1 November 2017

Accepted 26 December 2017

Available online 27 December 2017

\section{Keywords}

Magnetic systems

Ferromagnetic multilayers

Magnetization dynamics

Magnetoimpedance effect

\begin{abstract}
A B S T R A C T
We perform a theoretical and experimental investigation of the magnetic properties and magnetization dynamics of a ferromagnetic magnetostrictive multilayer grown onto a flexible substrate and submitted to external stress. We calculate the magnetic behavior and magnetoimpedance effect for a trilayered system from an approach that considers a magnetic permeability model for planar geometry and a magnetic free energy density which takes into account induced uniaxial and magnetoelastic anisotropy contributions. We verify remarkable modifications of the magnetic anisotropy with external stress, as well as we show that the dynamic magnetic response is strongly affected by these changes. We discuss the magnetic features that lead to modifications of the frequency limits where distinct mechanisms are responsible by the magnetoimpedance variations, enabling us to manipulate the resonance fields. To test the robustness of the approach, we directly compare theoretical results with experimental data. Thus, we provide experimental evidence to confirm the validity of the theoretical approach, as well as to manipulate the resonance fields to tune the MI response according to real applications in devices.
\end{abstract}

(c) 2017 Elsevier B.V. All rights reserved.

\section{Introduction}

Magnetoelastic properties in thin films and multilayers have attracted increasing attention in recent decade due to a wide variety of applications in magnetic memory elements [1-3] and acoustic generation of resonant spin-wave excitations [4,5]. Moreover, magnetostrictive films and multilayers grown onto flexible substrates appears as remarkable candidates as ground for spintronic devices, mainly due to the manipulable magnetic and mechanical properties [6-8]. This explains the recent interest in controlling and handling of properties as magnetic anisotropy, dynamic magnetic response, magnetostrictive properties and stress in ferromagnetic flexible nanostructures.

Nowadays, the progress in this field is highly driven by the emergence of magnetic or magnetoelectric devices operating at high frequencies $[9,10]$. However, irrespective on the future technological application, the study of the electrical and magnetic properties of magnetostrictive films and multilayers grown onto flexible substrates is essential. In this context, the comprehension of the mechanisms that control the magnetization dynamics is of fundamental importance to understand the basic physical

\footnotetext{
* Corresponding author.

E-mail addresses: marciocorrea@dfte.ufrn.br (M.A. Correa), felipebohn@fisica. ufrn.br (F. Bohn).
}

properties in these systems as well as to provide new roads to applications. Traditionally the main technique employed for the characterization of the dynamic magnetic response in nanostructures is the ferromagnetic resonance (FMR). In particular, FMR experiments may provide important information on the magnetic properties and high-frequency magnetic behavior in the saturated regime. On the other side, insights on the dynamic properties in the unsaturated magnetization regime are important to explore local anisotropies, mainly when their manipulation is the focus for technological application [8]. With this spirit, magnetoimpedance (MI) effect arises as an useful tool in the understanding of fundamental properties of the magnetization dynamics [11].

The MI effect corresponds to the change of the complex electric impedance $Z=R+i X$ of a ferromagnetic conductor submitted to an external magnetic field $H$. Thus, in a typical MI experiment, besides the external field, the sample is also submitted to an alternating magnetic field associated with the probe electric current $I_{a c}=I_{0} \exp (2 \pi f t)$, with $f$ being the current frequency. The MI effect is directly connected with the transverse magnetic permeability through the Skin effect, in which the drive current has a limited penetration thickness in the sample. Considering a ferromagnetic material, the transverse magnetic permeability can be manipulated by the frequency, external magnetic field, and magnetic state of the sample (saturated or unsaturated). Therefore, the control of these properties is a key issue to achieve good MI response in a 
given material. Besides, by choosing an appropriate material and structure, it is possible to tailor desired impedance responses, according to each specific technological application.

In this paper, we report a theoretical and experimental investigation of the magnetic properties and magnetization dynamics of a ferromagnetic magnetostrictive multilayer grown onto a flexible substrate and submitted to external stress. From numerical calculation, we address the stress dependence of the effective magnetic anisotropy and dynamical magnetic response of the multilayer. We verify remarkable modifications of the magnetic anisotropy with external stress, as well as we show that the dynamic magnetic response is strongly affected by these changes. To test the robustness of the approach, we corroborate our theoretical predictions by comparing them with experiments. We interpret the results in terms of the evolution of the effective magnetic anisotropy with the increase of the magnetoelastic contribution to the free energy density. Moreover, we discuss the magnetic features that lead to modifications of the frequency limits where distinct mechanisms are responsible by the MI variations, enabling us to manipulate the resonance fields to tune the MI response according to real applications in devices.

\section{Numerical calculation}

First of all, we perform numerical calculation of the quasi-static and dynamical magnetic properties in order to verify the influence of the stress on the effective magnetic anisotropy and magnetoimpedance effect. To this end, from the appropriate magnetic free energy density for the investigated structure, we consider a general magnetic susceptibility model, which takes into account its dependence with both frequency and magnetic field [12-14]. It is therefore possible to obtain the transverse magnetic permeability for planar geometry from susceptibility and in turn describe the MI behavior by using different models [15,16], according to system structure, for a wide range of frequencies and external magnetic fields.

Here, we focus on a ferromagnetic magnetostrictive multilayer grown onto a flexible substrate and submitted to external stress, and we model it as a planar system. Fig. 1 presents the theoretical system and the definitions of the relevant vectors considered to perform the numerical calculation. Thus, from the appropriate magnetic free energy density $\xi$, a routine for energy minimization determines the values the equilibrium angles $\theta_{M}$ and $\varphi_{M}$ of the magnetization for a given external magnetic field $\vec{H}$, and we obtain the magnetization curve, permeability tensor $\mu$, and magnetoimpedance $Z$ in a wide range of frequencies.

In the magnetic susceptibility model, the dynamics magnetization is governed by the Landau-Lifshitz-Gilbert equation, given by $[12,14]$

$\frac{d \vec{M}}{d t}=-\gamma\left(\vec{M} \times \vec{H}_{e f f}\right)-\gamma \frac{\alpha}{M}\left[\vec{M} \times\left(\vec{M} \times \vec{H}_{e f f}\right)\right]$,

where $\vec{M}$ is the magnetization vector, $\vec{H}_{\text {eff }}$ is the effective magnetic field, and $\gamma=\left|\gamma_{G}\right| /\left(1+\alpha^{2}\right)$, in which $\gamma_{G}$ is the gyromagnetic ratio and $\alpha$ the phenomenological Gilbert damping constant. In particular, the effective magnetic field is described by $\vec{H}_{e f f}=\vec{H}_{\xi}+\vec{h}_{a c}$, where $\vec{H}_{\xi}=-\frac{\partial \xi}{\partial \vec{M}}$, which contains the external magnetic field $\vec{H}$ [17] and the internal field due to different contributions to the magnetic free energy density $\xi$, and $\vec{h}_{a c}$ is the alternating magnetic field generated by the $I_{a c}$ current flowing in the sample.

We consider the magnetization vector in the plane of the film, which turns the magnetic permeability tensor terms considerably simplified. From now, the transverse magnetic permeability $\mu_{t}$ may be expressed as $[12,14]$



Fig. 1. Schematic diagram of the theoretical system and definitions of the vectors employed for the numerical calculation of magnetization, transverse magnetic permeability, and magnetoimpedance curves. Here we consider $\vec{M}$ as the magnetization vector, whose orientation for each given magnetic field value is set by $\theta_{M}$ and $\varphi_{M}$, the equilibrium angles with respect to the $z$ and $x$ axes, respectively. The $\vec{H}$ corresponds to the external magnetic field vector, which is applied along the $y$ direction, i.e. $\theta_{H}=\varphi_{H}=90^{\circ}$. The unit vector $\hat{u}_{k}$ indicates the direction of the uniaxial magnetic anisotropy induced during deposition, while $\hat{u}_{\sigma}$ presents the direction of the magnetoelastic anisotropy induced by external stress applied during the experiment. Finally, $\hat{u}_{\text {eff }}$ describes the orientation of the effective magnetic anisotropy, a result of the competition between the contributions of the induced uniaxial and magnetoelastic anisotropies. The unit vector normal to the plane of the film is along the $z$ direction, i.e. $\hat{n}=\hat{k}$.

$$
\begin{aligned}
\mu_{t}=1 & +4 \pi \kappa \sin ^{2} \varphi_{M} \times\left[\left(\omega_{r}^{2}-\omega^{2}\right)\left(1+\alpha^{2}\right) \gamma \xi_{\theta \theta}+\alpha M_{s} \omega^{2} \Delta \omega\right. \\
& \left.+i\left[-\left(1+\alpha^{2}\right) \gamma \omega \Delta \omega \xi_{\theta \theta}+\alpha M_{s} \omega\left(\omega_{r}^{2}-\omega^{2}\right)\right]\right],
\end{aligned}
$$

where $\kappa$ is defined as

$\kappa=\frac{\gamma}{\left(\omega_{r}^{2}-\omega^{2}\right)+\omega^{2} \Delta \omega^{2}}$

and $\omega_{r}$ and $\Delta \omega$ are respectively the well-known angular resonance frequency and width of the resonance absorption line, given by

$\omega_{r}=\frac{\gamma}{M \sin \theta_{M}} \sqrt{1+\alpha^{2}} \sqrt{\xi_{\theta \theta} \xi_{\varphi \varphi}-\xi_{\theta \varphi}^{2}}$,

and

$\Delta \omega=\frac{\alpha \gamma}{M}\left(\xi_{\theta \theta}+\frac{\xi_{\varphi \varphi}}{\sin ^{2} \theta_{M}}\right)$.

From the last three equations, $\xi_{\theta \theta}, \xi_{\varphi \varphi}, \xi_{\varphi \theta}$, and $\xi_{\theta \varphi}$ are the second derivatives of the magnetic free energy density at an equilibrium position, defined by the magnetization vector with $\theta_{M}$ and $\varphi_{M}$, as previously shown in Fig. 1 .

To describe the magnetization dynamics through the magnetoimpedance effect in a multilayer system, we consider the traditional approach to study the MI effect in a trilayered system reported by Panina et al. [16] and previously investigated by our group $[13,14]$. In this model, the trilayered system has finite width $2 b$ and length $l$ for all layers, thicknesses $t_{1}$ and $t_{2}$ and conductivity values $1 / \rho_{1}$ and $1 / \rho_{2}$ for the metallic non-magnetic and ferromagnetic layers, respectively. When $b$ is sufficiently large and the edge effect is neglected, impedance is dependent on the film thickness $t$. Therefore, for a trilayered system, the impedance can be written as $[16,14]$

$\frac{Z}{R_{d c}}=\left(\eta_{m} \eta_{f}\right)\left[\frac{\operatorname{coth}\left(\frac{\eta_{m} / \rho_{2}}{\mu_{t} / \rho_{1}}\right) \operatorname{coth}\left(\eta_{f}\right)+\frac{2 \eta_{m}}{k_{1} t_{1}}}{\operatorname{coth}\left(\frac{\eta_{m} / \rho_{2}}{\mu_{t} / \rho_{1}}\right)+\frac{2 \eta_{m}}{k_{1} t_{1}} \operatorname{coth}\left(\eta_{f}\right)}\right]$,

where 


$$
\begin{aligned}
& \delta_{1}=\left(2 \pi \omega / \rho_{1}\right)^{-1 / 2}, \quad \delta_{2}=\left(2 \pi \omega \mu_{t} / \rho_{2}\right)^{-1 / 2}, \\
& \eta_{m}=\frac{k_{1} t_{1}}{2}\left(\frac{\mu_{t} / \rho_{1}}{1 / \rho_{2}}\right), \quad \eta_{f}=k_{2} t_{2}, \\
& k_{1}=\frac{(1-i)}{\delta_{1}}, \quad k_{2}=\frac{(1-i)}{\delta_{2}} .
\end{aligned}
$$

To perform the numerical calculation of the transverse magnetic permeability (Eq. (2)) and magnetoimpedance effect (Eq. (6)) for a ferromagnetic magnetostrictive trilayered system, which has an induced uniaxial magnetic anisotropy and is submitted to external stress, we consider the following free magnetic energy density

$$
\xi=\sum_{i=1}^{2}\left[-\vec{M}_{i} \cdot \vec{H}+4 \pi M_{s i}^{2}\left(\widehat{M}_{i} \cdot \widehat{n}\right)-\frac{H_{k i}}{2 M_{s i}}\left(\widehat{M}_{i} \cdot \widehat{u}_{k i}\right)^{2}-\frac{3}{2} \lambda_{s_{i}} \sigma_{i}\left(\widehat{M}_{i} \cdot \widehat{u}_{\sigma_{i}}\right)^{2}\right] \text {, }
$$

where $\vec{M}_{i}$ and $M_{s i}$ are the magnetization vector and saturation magnetization for each ferromagnetic layer, $H_{k i}=2 K_{u i} / M_{s i}$ is the anisotropy field for each layer, $K_{u i}$ is the respective induced uniaxial magnetic anisotropy constant, $\lambda_{s i}$ is the saturation magnetostriction constant for each ferromagnetic layer, and $\sigma_{i}$ is the external stress. For sake of simplicity, in theory, it is reasonable to assume that $\vec{M}_{1}=\vec{M}_{2}=\vec{M}_{s} \quad$ (consequently $\left.\quad M_{s_{1}}=M_{s_{2}}=M_{s}\right), \quad H_{k_{1}}=H_{k_{2}}=H_{k}$, $\widehat{u}_{k_{1}}=\widehat{u}_{k_{2}}=\widehat{u}_{k}, \quad \lambda_{s_{1}}=\lambda_{s_{2}}=\lambda_{s}, \quad \sigma_{1}=\sigma_{2}=\sigma, \quad$ and finally, $\widehat{u}_{\sigma_{1}}=\widehat{u}_{\sigma_{2}}=\widehat{u}_{\sigma}$, since the two layers are made of the same ferromagnet and share similar magnetic properties.

The first term of the free magnetic energy density indicates the Zeeman interaction, the second one is the demagnetizing energy density, and the third term corresponds to the induced uniaxial magnetic anisotropy oriented along $\widehat{u}_{k}$. Finally, the fourth term describes the magnetoelastic energy density for an elastically isotropic medium with isotropic magnetostricion. This term relates the saturation magnetostriction constant $\lambda_{s}$ and the external stress $\sigma$ applied to the system along $\widehat{u}_{\sigma}$. In particular, the product between $\lambda_{s}$ and $\sigma$ induced by the stress application modifies the effective magnetic anisotropy of the sample. In this case, if $\lambda_{s} \sigma>0$, an anisotropy axis is induced along the same direction of the applied external stress. On the other hand, if $\lambda_{s} \sigma<0$, this anisotropy axis is oriented perpendicular to the direction of external stress $[18,8]$.

For the numerical calculation, we consider as system parameters: $M_{s_{1}}=M_{s_{2}}=1080 \mathrm{emu} / \mathrm{cm}^{3}$ and $\lambda_{s}=+26 \times 10^{-6}$, characteristic values of the $\mathrm{Fe}_{73.5} \mathrm{Cu}_{1} \mathrm{Nb}_{3} \mathrm{Si}_{13.5} \mathrm{~B}_{9}$ alloy [19], $H_{k_{1}}=H_{k_{2}}=15$ Oe, $\theta_{k_{1}}=\theta_{k_{2}}=90^{\circ}, \varphi_{k_{1}}=\varphi_{k_{2}}=5^{\circ}, \alpha=0.07, \gamma_{G} / 2 \pi=3.038 \mathrm{MHz} / \mathrm{Oe}$, $1 / \rho_{1}=6 \times 10^{7}(\Omega \mathrm{m})^{-1}$, and $1 / \rho_{2}=1 /\left(4 \rho_{1}\right), t_{1}=2 \mathrm{~nm}$, and $t_{2}=10 \mathrm{~nm}$. In particular, the thickness of the metallic nonmagnetic layer is thick enough to neglect the bilinear and biquadratic coupling between the ferromagnetic layers. Besides, as indicated in Fig. 1, we assume the following experimental parameters: magnetic field oriented with $\theta_{H}=90^{\circ}$ and $\varphi_{H}=90^{\circ}$, while external stress applied with $\theta_{\sigma}=90^{\circ}$, and $\varphi_{\sigma}=90^{\circ}$.

Fig. 2 shows the numerical calculation of the magnetization curves for the magnetostrictive trilayered system under different external stress values. The curve obtained for the system without external stress (black solid line in Fig. 2) confirms the induced in-plane magnetic anisotropy, with easy magnetization axis perpendicular to the orientation of the magnetic field, as expected. The small value for $\varphi_{k}=5^{\circ}$ infers an hysteretic behavior, given rise to a typical hard axis behavior with non-zero coercive field.

The magnetic properties are strongly dependent on the external stress. With increasing the stress, it is observed a clear evolution of the shape of the magnetization curve, from an inclined and narrowed curve (red solid line) to a squared shaped loop (blue



Fig. 2. Normalized magnetization curves calculated for the magnetic system under different external stress values. The numerical calculation are obtained for a system with $M_{s_{1}}=M_{s_{2}}=1080 \mathrm{emu} / \mathrm{cm}^{3} \quad$ and $\lambda_{s}=+26 \times 10^{-6}, \quad H_{k_{1}}=H_{k_{2}}=15 \mathrm{Oe}$ $\theta_{k_{1}}=\theta_{k_{2}}=90^{\circ}, \quad \varphi_{k_{1}}=\varphi_{k_{2}}=5^{\circ}, \quad \alpha=0.07, \quad \gamma_{G} / 2 \pi=3.038 \mathrm{MHz} / \mathrm{Oe}$, $1 / \rho_{1}=6 \times 10^{7}(\Omega \mathrm{m})^{-1}$, and $1 / \rho_{2}=1 /\left(4 \rho_{1}\right), t_{1}=2 \mathrm{~nm}, t_{2}=10 \mathrm{~nm}, \theta_{H}=90^{\circ}$, $\varphi_{H}=90^{\circ}, \theta_{\sigma}=90^{\circ}$, and $\varphi_{\sigma}=90^{\circ}$.

solid line). This is a consequence of the raise of the magnetoelastic anisotropy contribution to the effective magnetic anisotropy. Given that the saturation magnetostriction constant is positive, a magnetoelastic anisotropy axis arises along the direction of the positive external stress, i.e. in this case perpendicular to the uniaxial anisotropy and along the same orientation of the magnetic field. Notice that the calculations are performed with $\widehat{u}_{k} \perp \vec{H}$, and $\widehat{u}_{\sigma} \| \vec{H}$. It leads to changes in the orientation $\widehat{u}_{\text {eff }}$ of the effective magnetic anisotropy with the increase of the stress value, thus modifying the resulting whole magnetic behavior and magnetization curves. For $\sigma=65 \mathrm{MPa}$, the squared curve with smaller coercive field reveals an intermediate magnetic behavior, arisen from an effective magnetic anisotropy, with smaller magnitude, oriented very close to the direction stress and magnetic field. However, for $\sigma=125 \mathrm{MPa}$, the external stress is high enough to set the effective magnetic anisotropy axis parallel to the stress and magnetic field. Consequently, the magnetization curve has fingerprints of an easy magnetization axis.

Thus, the magnetic behavior of the magnetostrictive trilayered system under different external stress values is a result of the competition between the induced uniaxial magnetic anisotropy and the magnetoelastic anisotropy contribution. In this sense, this competition allows us to manipulate the magnetic anisotropy and, consequently, the magnetoimpedance. Moreover, specially for the MI effect, the orientation between the external magnetic field and ac current with respect to the effective anisotropy defines the mechanisms responsible by the MI variations, as well as influences the frequency limits where the FMR effect acts as the main mechanism changing the electrical impedance. As a consequence, the peaks structure of the magnetoimpedance curves is modified according to effective magnetic anisotropy [20].

Fig. 3 shows the numerical calculation of the relation between resonance frequency $f_{r}$ and external magnetic field $H$, i.e. the dispersion relation, when the system is submitted to different external stress values. 


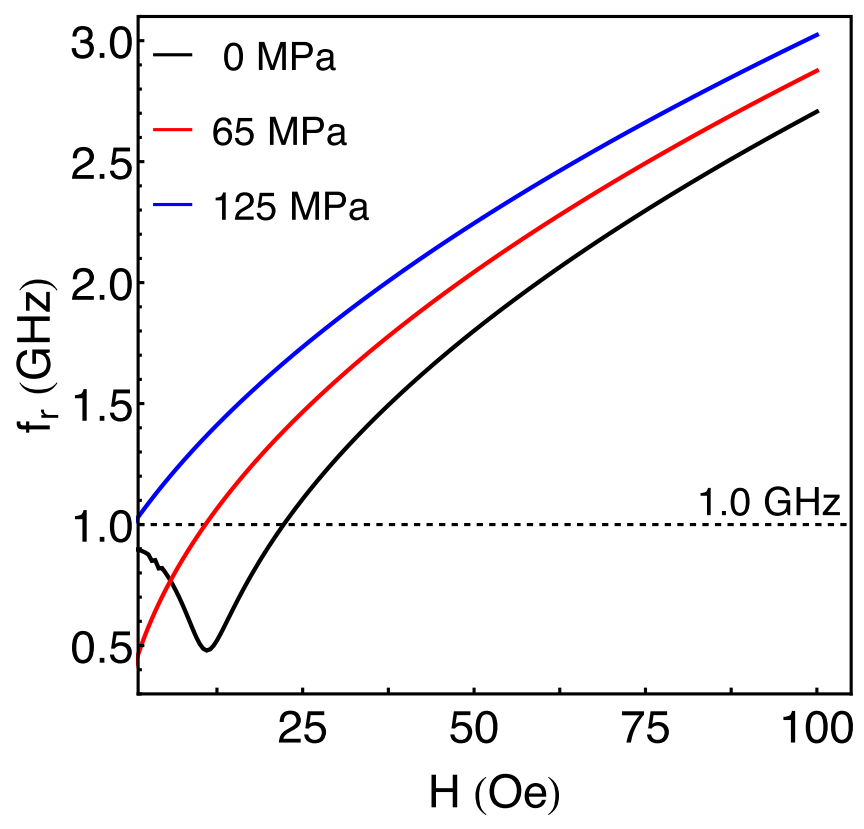

Fig. 3. Numerical calculation of the dispersion relation for different external stress values. The calculation is performed using parameters similar to those previously employed. The dashed line indicates the frequency of $1.0 \mathrm{GHz}$.

For the system without external stress, the magnetic fields configuration employed here favors the alignment between $\vec{h}_{a c}$, i.e. the field generated by the ac current, and the induced uniaxial magnetic anisotropy oriented along $\widehat{u}_{k}$. In this case, $f_{r}$ has a minimum located close to the anisotropy field $H_{k}$ (black solid line in Fig. 3 ). Moreover, above $\sim 1.0 \mathrm{GHz}$, just one resonance positive field is verified for a given resonance frequency $f_{r}$, leading to a double peak structure in the MI curves. Below this frequency, there is a range where two positive magnetic field values satisfy the resonance condition, leading in principle to a multiple peak structure [20].

On the other hand, with increasing the stress and the consequent modification in the orientation and intensity of the effective anisotropy, a condition in which the magnetic field lies along $\widehat{u}_{\text {eff }}$, and consequently $\vec{h}_{a c}$ is perpendicular to this effective anisotropy, is achieved.

Thus, just one resonance positive field is verified for each resonance frequency $f_{r}$, leading to a double peak structure in the MI curves. Moreover, in particular, $f_{r}$ has a minimum at $H \approx 0$. However, notice that the frequency value, where the minimum is identified, is strongly dependent on the effective magnetic anisotropy. Here, for this fields configuration, the higher the external stress and consequently the magnetoelastic anisotropy, the higher the frequency in which the FMR effect starts appearing.

Fig. 4 presents the numerical calculation for the real $R$ component of the impedance as a function of the external magnetic field, for selected external stress values, when the system is at different frequencies. The curves are presented only for positive magnetic field values, although this behavior is also observed for negative field values. The electric contribution of the impedance is not considered here and just the magnetic contribution of the impedance is shown.

For the system without external stress, the double peak structure is found for the whole frequency range, from $1.0 \mathrm{GHz}$, following the dispersion relation. With increasing the frequency, the displacement of the $R$ peak towards higher magnetic field values is a signature of the FMR effect governing the magnetization dynamics. On the other hand, for $\sigma=125 \mathrm{MPa}$, a curve characteristic of the parallel alignment between $\vec{h}_{a c}$ and $\widehat{u}_{\text {eff }}$ is observed. The $R$ curve calculated at $1.0 \mathrm{GHz}$ has a single peak structure, with the peak located at $H \sim 0$, indicating that the skin effect governs the magnetization dynamics at this frequency range. For frequencies above this value, besides the skin effect, the FMR effect also becomes an important mechanism responsible for MI variations, a fact evidenced through the change of the peaks structure, as well as to the displacement in field of the peaks as the frequency is raised. Similar behavior is found for $\sigma \sim 65 \mathrm{MPa}$, in which the FMR features are already observed from $\sim 0.5 \mathrm{GHz}$.

Our results raise an interesting issue on the MI behavior in magnetostrictive multilayers and the possibility of manipulating the magnetic anisotropy and magnetization dynamics by the external stress. Fig. 5 presents the numerical calculation for the real $R$ component of the impedance as a function of the external magnetic field, for selected frequencies, when the magnetostrictive trilayered system is submitted to different external stress values. Here, the external stress is responsible by both, the displacement of the peaks in field, as well as the evolution of the magnetoimpedance peaks structure. At $1.0 \mathrm{GHz}$, the double peak structure found for the system without external stress evolves as the stress increases, giving rise to a single peak structure when the external stress reaches $125 \mathrm{MPa}$, as expected. The changes of the effective magnetic anisotropy occurring as the external stress is varied, in association with the changes in its orientation with
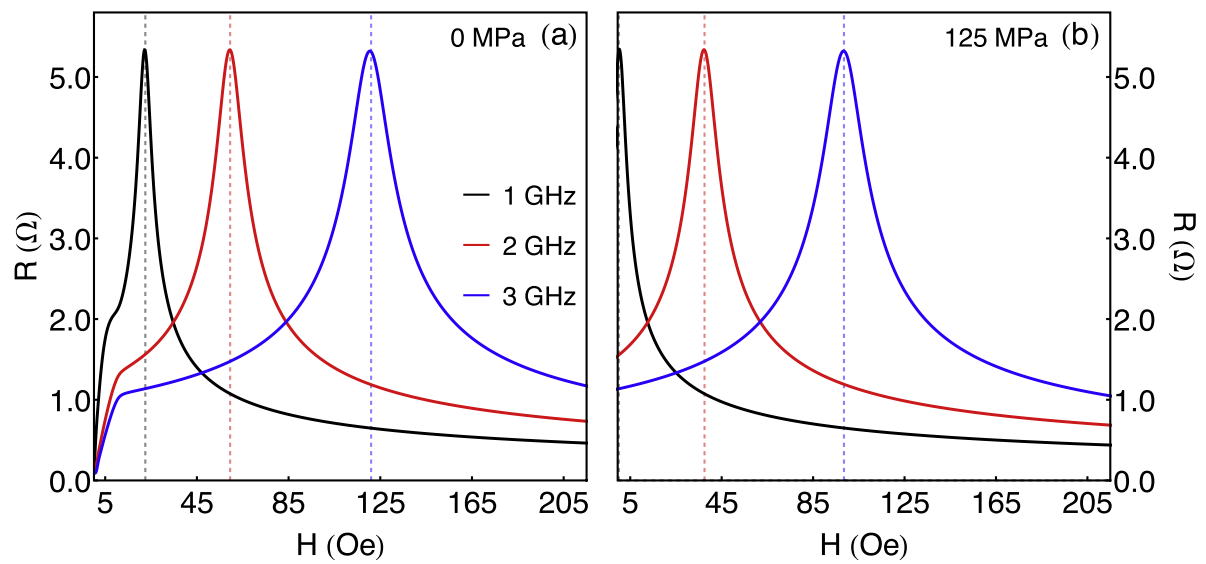

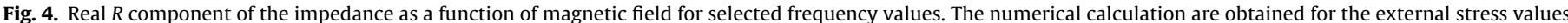

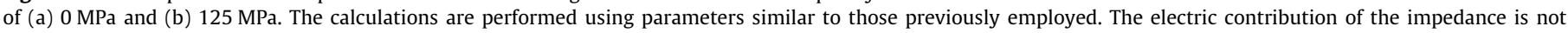
considered here and just the magnetic contribution of the impedance is shown. 

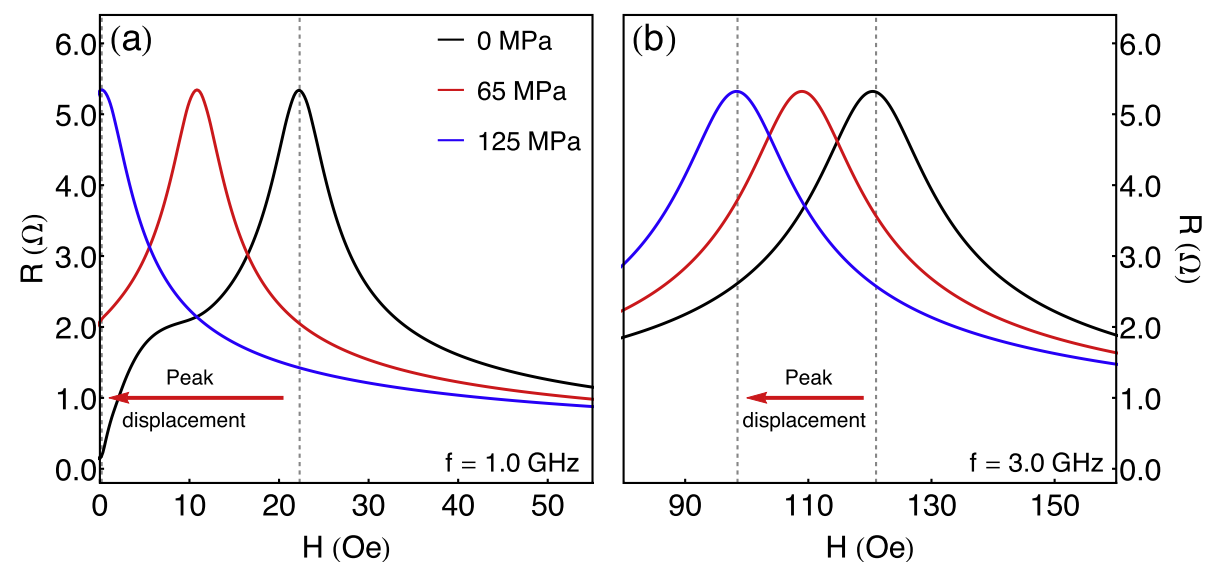



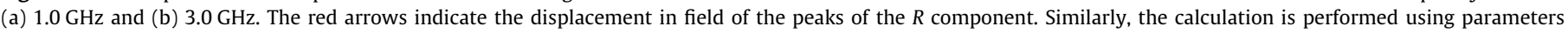

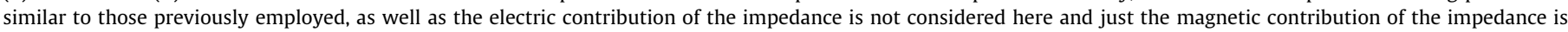
shown.

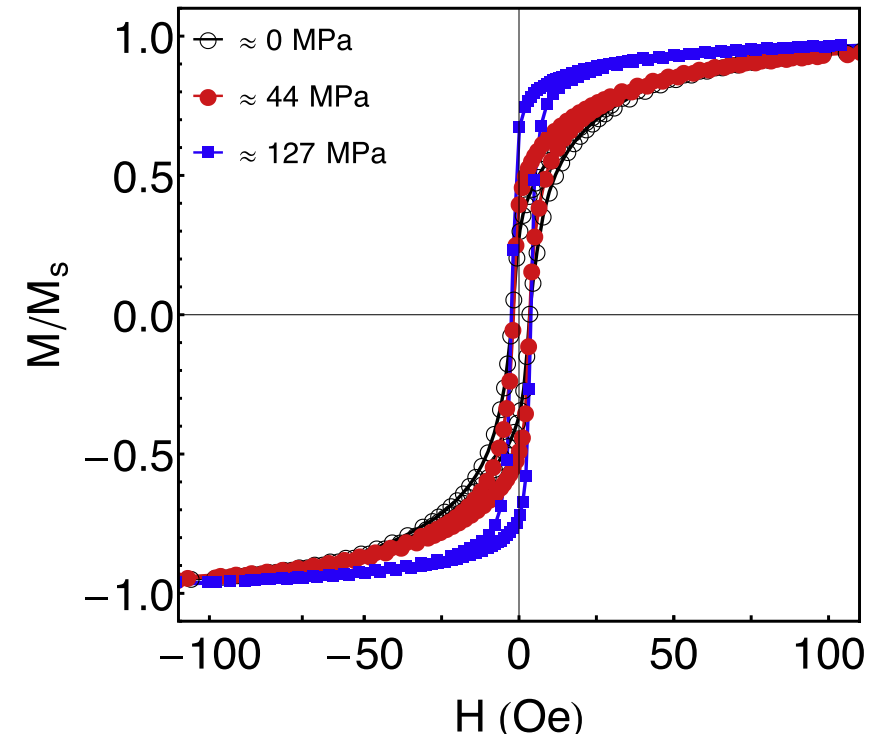

Fig. 6. Normalized experimental magnetization curves measured for the FeCuNb$\mathrm{SiB} / \mathrm{Ta}$ multilayer under different external stress values. The curves are acquired with magnetic field applied along the longer axis of the multilayer, i.e. perpendicular to the easy magnetization axis induced during deposition, and parallel to direction of the external stress.

respect to the fields configuration, induces modifications in the mechanism governing the magnetization dynamics, from FMR to Skin effect. On the other hand, at the high frequency range where the FMR effect is mechanism responsible by the MI variations irrespectively on the effective magnetic anisotropy, the double peak structure is kept as the stress increases, although a displacement in field of peaks is observed. Thus, the influence of the external stress on the magnetic anisotropy and magnetization dynamics makes possible the tuning of resonance fields, as well as the whole MI response.

\section{Comparison with the experiment}

The previous results obtained with the theoretical approach have described qualitatively the main features of a magnetostrictive multilayer submitted to external stress. To verify the validity of the theoretical approach, we investigate the quasi-static and dynamical magnetic properties of a ferromagnetic magnetostrictive $\left[\mathrm{Fe}_{73.5} \mathrm{Cu}_{1} \mathrm{Nb}_{3} \mathrm{Si}_{13.5} \mathrm{~B}_{9}(10 \mathrm{~nm}) / \mathrm{Ta}(2 \mathrm{~nm})\right] \times 50$ multilayer grown onto a flexible Kapton ${ }^{\circledR}$ substrate, submitted to external stress, and compare the experimental results with the numerical calculation. In particular, the ferromagnetic alloy has high saturation magnetostriction constant of $\lambda_{s} \approx+26 \times 10^{-6}$ [21,19]. The multilayer has dimensions of $10 \times 4 \mathrm{~mm}^{2}$. The samples are produced by magnetron sputtering, with the following parameters [8]: base pressure of $7 \times 10^{-7}$ Torr, deposition pressure of $2 \times 10^{-3}$ Torr with Ar at $32 \mathrm{sccm}$ constant flow, and using a DC source with $20 \mathrm{~W}$. During the deposition, a constant in-plane $1 \mathrm{kOe}$ magnetic field is applied perpendicularly to the longer axis of the substrate to induce a magnetic anisotropy and define an easy magnetization axis. Magnetic behavior at room temperature is obtained by magnetization curves acquired with a vibrating sample magnetometer. The magnetoimpedance effect experiments are carried out with a RF-impedance analyzer Agilent model 4991, with E4991A test head connected to a microstrip in which the sample is the central conductor. The real $R$ and imaginary $X$ components of the impedance are acquired in a wide frequency range, from 0.5 up to 3.0 $\mathrm{GHz}$. The measurement are obtained at $0 \mathrm{dBm}(1 \mathrm{~mW})$ constant power, characterizing a linear regime of driving signal. For the experiments, the effective magnetic anisotropy is modified by the external stress by bending the flexible substrate. The stress is estimated following the approach described in Refs. [22,23], which is based on the Stoney model. In particular, the $\sigma$ values considered here are $\approx 0, \approx 44$, and $\approx 127 \mathrm{MPa}$. Further details on the sample and the experiments are provided in Ref. [8]. Thus, the complexity of the considered system, including different features previously studied, and the quantitative agreement with experimental results do confirm the robustness of our theoretical approach.

Fig. 6 shows the normalized magnetization curves obtained for the magnetostrictive multilayer. Experimental magnetization curves are obtained with the sample under different external stress values. Notice the evolution of the magnetization curves with the increase of the stress, from an inclined, narrowed curve, characteristic of a hard magnetization axis, to a near squared shaped loop, evidencing an easy magnetization axis. These features are in concordance with the modifications of the effective magnetic anisotropy predicted by our theoretical approach and shown in Fig. 2.

The quasi-static magnetic properties play a fundamental role in the dynamic magnetic response of a ferromagnetic system. Thus, the changes in the magnetic anisotropy and magnetization curves are reflected in the magnetoimpedance curves. Regarding the 


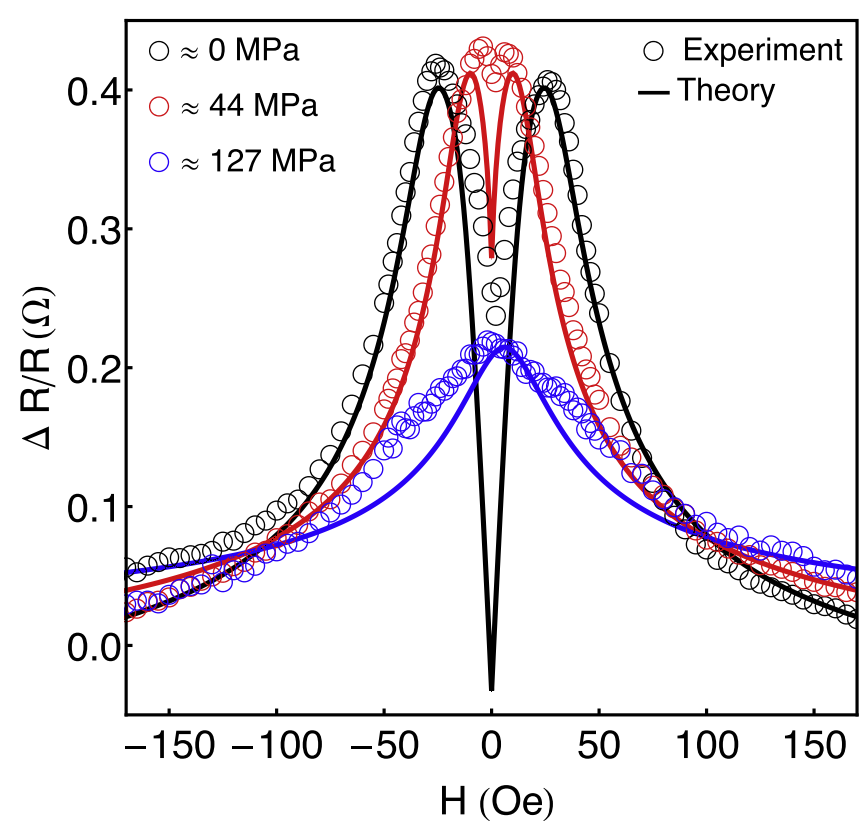

Fig. 7. Experimental results and numerical calculation of $\Delta R / R$, at $1.0 \mathrm{GHz}$, as a function of the magnetic field for selected external stress values.

results, in order to allow direct comparison between theory and experiment, we define

$\Delta R / R=\left[R(H)-R\left(H_{\max }\right)\right] / R\left(H_{\max }\right)$,

where $R(H)$ is the real component of the impedance at a given external magnetic field and $R\left(H_{\max }\right)$ is its value for the maximum external magnetic field, where the multilayer is saturated magnetically.

Fig. 7 presents experimental data and numerical calculation for $\Delta R / R$, at $1.0 \mathrm{GHz}$, as a function of the magnetic field for selected external stress values. For all cases, it is evident the quantitative agreement between the experimental data and numerical calculation. In particular, the calculation performed using similar parameters previously employed are able to describe all the main features of magnetic behavior for each external stress value. However, considering the quasi-static magnetic behavior of the multilayer, small changes in the magnetic parameters, such as $\varphi_{k} \approx 25^{\circ}, H_{k} \approx 80$ Oe and the aforementioned $\sigma$ values, are needed for the calculation. At the same time, to tune the amplitude of the curves, we slightly modify the $R_{d c}$ value of the experimental sample for each case, following the procedure described in Ref. [14].

The tiny differences between experiment and theory, specially at low fields, may be devoted to the magnetic domain structure, which is not taken into account in our model. At the same time, at higher fields where the domain structure is suppressed, discrepancies are not found. From a general point of view, the change of the peaks structure as well as the location of the resonance peaks, both related with modifications of the effective magnetic anisotropy, are experimentally observed.

By considering magnetostrictive multilayers, the best $\mathrm{MI}$ response can be explored by playing with sample's parameters, such as substrate, type of structure, thickness and composition of the ferromagnetic material, the latter directly related to magnetic properties as magnetostriction, and experimental parameters. Thus, the most striking finding here resides in the MI variations tuned by modifying the magnetic field, frequency, magnetostriction, and external stress.

\section{Conclusion}

In conclusion, we have performed a theoretical and experimental investigation of the magnetic properties and magnetization dynamics of a ferromagnetic magnetostrictive multilayer grown onto a flexible substrate and submitted to external stress. From numerical calculation using an approach that considers a magnetic permeability model for planar geometry and an magnetic free energy density which takes into account induced uniaxial and magnetoelastic anisotropy contributions, we have addressed the stress dependence of the magnetic anisotropy and dynamical magnetic response of the multilayer. We have verified remarkable modifications of the magnetic anisotropy with external stress, as well as we have shown that the dynamic magnetic response is strongly affected by these changes. The theoretical approach has enabled us to infer the relation between effective anisotropy and the mechanisms that govern the magnetization dynamics in magnetostrictive materials. To test the robustness of the approach, we have compared our theoretical predictions with experiments. In this case, the agreement between theory and experiment has confirmed the validity of the theoretical approaches, as well as has provided further insights on the magnetization dynamics in ferromagnetic magnetostrictive multilayer grown onto a flexible substrate and submitted to external stress, enabling us to manipulate the resonance fields to tune the MI response according to real applications in devices.

\section{Acknowledgments}

The research is supported by the Brazilian agencies $\mathrm{CNPq}$ (Grants No. 306423/2014-6, No. 306362/2014-7, and No. 441760/2014-7), CAPES, and FAPERN.

\section{References}

[1] A. Ludwig, E. Quandt, J. Appl. Phys. 87 (2000) 4691-4695.

[2] P.G. Gowtham, D. Labanowski, S. Salahuddin, Phys, Rev, B 94 (2016), 014436.

[3] L. Thevenard, I.S. Camara, J.-Y. Prieur, P. Rovillain, A. Lemâtre, C. Gourdon, J.-Y. Duquesne, Phys. Rev. B 93 (2016) 140405.

[4] N.I. Polzikova, S.G. Alekseev, I.I. Pyataikin, I.M. Kotelyanskii, V.A. Luzanov, A.P. Orlov, AIP Adv. 6 (2016), 056306.

[5] D. Labanowski, A. Jung, S. Salahuddin, Appl. Phys. Lett. 108 (2016), 022905.

[6] O. Lee, L. You, J. Jang, V. Subramanian, S. Salahuddin, Appl. Phys. Lett. 107 (2015) 252401.

[7] M. Gueye, B.M. Wague, F. Zighem, M. Belmeguenai, M.S. Gabor, T. Petrisor, C. Tiusan, S. Mercone, D. Faurie, Appl. Phys. Lett. 105 (2014), 062409.

[8] K. Agra, F. Bohn, T. Mori, G. Callegari, L. Dorneles, M. Correa, J. Magn. Magn. Mater. 420 (2016) 81-87.

[9] P. Hayes, S. Salzer, J. Reermann, E. Yarar, V. Robisch, A. Piorra, D. Meyners, M. Hoft, R. Knochel, G. Schmidt, E. Quandt, Appl. Phys. Lett. 108 (2016), https:// doi.org/10.1063/1.4948470.

[10] M. Pernpeintner, R.B. Holländer, M.J. Seitner, E.M. Weig, R. Gross, S.T.B. Goennenwein, H. Huebl, J. Appl. Phys. 119 (2016), 093901.

[11] M.-H. Phan, H.-X. Peng, Prog. Mater. Sci. 53 (2008) 323-420.

[12] L. Spinu, I. Dumitru, A. Stancu, D. Cimpoesu, J. Magn. Magn. Mater. 296 (2006) $1-8$.

[13] M.A. Corrêa, F. Bohn, V.M. Escobar, M.S. Marques, A.D.C. Viegas, L.F. Schelp, R.L. Sommer, J. Appl. Phys. 110 (2011), 093914.

[14] M.A. Corrêa, F. Bohn, R.B. da Silva, R.L. Sommer, J. Appl. Phys. 116 (2014) 243904.

[15] L. Kraus, J. Magn. Magn. Mater. 195 (1999) 764-778.

[16] L. Panina, K. Mohri, Sens. Act. A. 81 (2000) 71-77.

[17] J. Smit, H.G. Beljers, Philips Res. Rep. 10 (1955) 113.

[18] B.D. Cullity, Introduction to Magnetic Materials, Addison-Wesley, New York, 1972.

[19] G. Herzer, J. Magn. Magn. Mater. 112 (1992) 258.

[20] A.M.H. de Andrade, M.A. Corrêa, A.D.C. Viegas, F. Bohn, R.L. Sommer, J. Appl. Phys. 115 (2014) 103908.

[21] P. Sharma, A. Gupta, J. Magn. Magn. Mater. 288 (2005) 347.

[22] C.-H. Hsueh, J. Appl. Phys. 91 (2002) 9652-9656.

[23] Y.Y. Hu, W.M. Huang, J. Appl. Phys. 96 (2004) 4154-4160. 